\title{
Einführung von Online Academy Campuses und Classrooms
}

Nur 1 Jahr nach der erfolgreichen Lancierung seiner E-Learning-Plattform, der ITI Online Academy, kündigte das Internationale Team für Implantologie (ITI), mit der Einführung der Campuses und Classrooms eine bedeutende Erweiterung der ITI Online Academy an. Diese erlauben es dem ITI zukünftig, besser auf die individuellen Fortbildungsbedürfnisse von Partnerorganisationen und Dritten einzugehen, indem sie es diesen ermöglichen, eigene Lernumgebungen auf der ITI Online Academy einzurichten. Die Campuses und ihre Classrooms sind ein ausgezeichnetes Mittel, um den Präsenzunterricht zu ergänzen und Lerngruppen, die curriculare Kurse besuchen, Lernmaterialien und -dienstleistungen anzubieten. Dozenten können ihren Kursteilnehmern in einem Classroom Lerninhalte der Academy für die Kursnachbereitung sowie Vorablektüren und im Unterricht behandeltes Material zur Verfügung stellen. Gezielte Assessments ermöglichen die Überwachung der Lernfortschritte der Teilnehmer. Die Teilnehmer ihrerseits haben auch die Möglichkeit, via Case Cloud und in geschlossenen Fo- ren eigene Beiträge zu teilen und zu diskutieren. Jede ITI-Sektion wird z. B. ihren eigenen Campus erhalten, auf dem Fachleute Zugang zu sprachspezifischen Inhalten haben, z. B. zu an nationalen ITI Kongressen aufgezeichneten Vorträgen. Mit der vollständigen Eingliederung der ITI Study Clubs wird die derzeit kleine Anzahl an Pilot-Classrooms schnell wachsen: Bis zum Jahresende wird der Online Academy Campus mehr als 650 individuelle Lerngemeinschaften beheimaten - ein globales Netzwerk für integrierte, evidenzbasierte Fortbildung. „Eines der Hauptziele des ITI ist es, der führende akademische Anbieter von evidenzbasierter Fortbildung auf dem Gebiet der dentalen Implantologie zu werden, “sagte Prof. Dr. David Cochran, der Präsident des ITI, „die ITI Online Academy Campuses bieten eine solide Grundlage für integrierte Lernangebote und stellen deshalb einen weiteren bedeutenden Schritt zur Erreichung dieses Ziels dar."

Nach einer Pressemitteilung von ITI International Team for Implantology www.iti.org 